\title{
THE DENSITY OF ABELIAN CUBIC FIELDS
}

\section{HARVEY COHN ${ }^{1}$}

In the following note we show that the abelian cubic fields are rare in relation to all cubic fields over the rationals. This is no surprise since an irreducible cubic equation generates an abelian field if and only if the coefficients of the equation are subject to a certain restriction (that the discriminant of the equation be a perfect square). To express this rareness in analytic form we arrange the fields in order of increasing field-discriminant and we define

$N_{A}(x)=$ number of abelian cubic fields of (positive) discriminant $\leqq x$, and

$N(x)=$ number of (abelian and non-abelian) cubic fields of discriminant $\leqq x$ in absolute value.

Then we show by means of a "zeta-function discussion" that

$$
N_{A}(x) \sim(11 c / 18) x^{1 / 2},
$$

as $x \rightarrow \infty$,

where $c$ is given in formula (8) below. This result complements unpublished results kindly communicated by Davenport, to the effect that $N(x)=O(x)$, and by Heilbronn, to the effect that $N(x)>c_{1} x$ (even with the restriction that the fields be totally real).

It can be shown by class-field theory $[1]^{2}$ that abelian cubic fields of discriminant $f^{2}$ are enumerated as follows: There is a single field with $f=9$ or $f=q_{0}$ and there are for $\nu \geqq 1$ exactly $2^{\nu}$ fields with $f$ $=q_{0} \cdots q_{\nu}$ or $f=9 q_{1} \cdots q_{\nu}$, where the $q_{i}$ are distinct primes $\equiv 1$ (mod 6). (These fields are in fact subfields of the field of the $f$ th roots of unity.) Thus,

$$
N_{A}(x)=U\left(x^{1 / 2}\right) / 2+U\left(x^{1 / 2} / 9\right)-1 / 2
$$

where

$$
U(y)=\sum_{n \leqq y} u(n)
$$

and $u(n)$ is, in turn, described by the generating function:

$$
\phi(s)=\prod\left(1+2 q^{-s}\right)=\sum_{1}^{\infty} u(n) n^{-s},
$$

Presented to the Society, April 25, 1953; received by the editors April 22, 1953.

1 Research sponsored by the U.S. Army Office of Ordnance Research, under contract DA-20-018-ORD-12332.

2 Numbers in brackets are items cited in the bibliography at the end. 
defined (at first) for $\operatorname{Re} s>1$, where $q$ are the primes $\equiv 1(\bmod 6)$.

To estimate $U(y)$ we introduce the primes $r \equiv-1(\bmod 6)$ and start with the identity:

$$
\phi(s)=\frac{\Pi\left\{\left(1+2 q^{-s}\right)\left(1-q^{-s}\right)^{2}\right\} \prod\left\{1-r^{-2 s}\right\}}{\prod\left(1-q^{-s}\right) \prod\left(1-r^{-s}\right) \prod\left(1-q^{-s}\right) \prod\left(1+r^{-s}\right)} .
$$

We note that each product of "curly brackets," $\Pi\{\cdots\}$, is absolutely convergent for at least $\operatorname{Re} s>1 / 2$, while

$$
\left[\Pi\left(1-q^{-s}\right) \Pi\left(1-r^{-s}\right)\right]^{-1}=\zeta(s)\left(1-2^{-s}\right)\left(1-3^{-s}\right) .
$$

Moreover,

$$
L(s)=\sum_{1}^{\infty}(-3 / n) n^{-s}=\left(1+2^{-s}\right)^{-1}\left[\Pi\left(1-q^{-s}\right) \Pi\left(1+r^{-s}\right)\right]^{-1}
$$

is a convergent series for $\operatorname{Re} s>0$, with the special value $L(1)=\pi 3^{-3 / 2}$ by Dirichlet's formula for quadratic class number [2, p. 373]. Hence from the known behavior of $\zeta(s)$, it follows that the difference $\phi(s)$ $-c /(s-1)$ is analytic at $s=1$, where (using $\zeta(2)=\pi^{2} / 6$ ) we easily find

$$
c=\frac{3^{1 / 2}}{2 \pi} \Pi \frac{(q+2)(q-1)}{q(q+1)}=.28 \cdots .
$$

As before, $q$ are the primes $\equiv 1(\bmod 6)$. Now from the behavior of $\phi(s)$ at $s=1$, together with the behavior of $\zeta(s)$ in the critical strip, we can show that

$$
U(y) \sim c y \quad(\text { as } y \rightarrow \infty) .
$$

We omit the details since Landau's Handbuch presents them not only for the prime number theorem [3, pp. 180-193] but also for a problem even involving a similar generating function [3, pp. 649-660]. Formula (1) finally follows through the use of formula (2). Q.E.D.

\section{BIBLIOGRAPHY}

1. H. Hasse, Arithmetische Bestimmung von Grundeinheit und Klassenzahl in zyklischen, kubischen und biguadratischen Zahlkörpern, Berlin, 1950, p. 10.

2. - Vorlesungen über Zahlentheorie, Berlin, 1950. 1953.

3. E. Landau, Handbuch der Lehre von der Verteilung der Primzahlen, New York,

WAYNE UNIVERSITY 